\title{
Symmetric Minkowski planes ordered by separation
}

\author{
Helmut Karzel, Jarosław Kosiorek and Andrzej Matraś
}

\author{
Dedicated to Mario Marchi
}

\begin{abstract}
In Karzel et al. (J. Geom. 99:116-127, 2009) we introduced for a symmetric Minkowski plane $\mathfrak{M}:=\left(P, \Lambda, \mathfrak{G}_{1}, \mathfrak{G}_{2}\right)$ an order concept by the notion of an orthogonal valuation for the circles of $\Lambda$ and showed that there is a one to one correspondence between the valuations and the halforderings of the accompanying commutative field. Here we consider an order concept which is based on the notion of separation for quadruples of concyclic points and establish the connections between these two notions. Our main result (cf. Theorem 3.3) states that these concepts are equivalent.
\end{abstract}

Mathematics Subject Classification (2010). 51B20(2000).

Keywords. Minkowski planes, order, valuation, separation.

\section{Introduction}

Order concepts in geometry are usually based on both, points and blocks. Minkowski planes belong to the chain structures and so there appear two types of blocks, generators and chains latter also called circles (in the case of Minkowski planes). In [1] we showed that in a symmetric Minkowski plane $\mathfrak{M}:=\left(P, \Lambda, \mathfrak{G}_{1}, \mathfrak{G}_{2}\right)$ an order structure can be introduced where only the set $\Lambda$ of circles is involved, namely by a valuation which associates to each circle $C \in \Lambda$ a value $[C]$ which is 1 or -1 . Then, since $\Lambda$ can be endowed with a multiplication "." such that $(\Lambda, \cdot)$ becomes a group, it turns out that the valuation is a homomorphism of $(\Lambda, \cdot)$ in the cyclic group $(\{1,-1\}, \cdot)$ of order two hence $[A \cdot B]=[A] \cdot[B]$ for $A, B \in \Lambda$. Furthermore to a symmetric Minkowski plane $\mathfrak{M}$ there corresponds a commutative field $(\mathbf{F},+, \cdot)$ such that $(\Lambda, \cdot)$ is isomorphic to the projective linear group $P G L(2, \mathbf{F})$ and if [ ] is an orthogonal valuation of 
$\mathfrak{M}$ then there is a halfordering $\eta$ of the commutative field $(\mathbf{F},+, \cdot)$ such that if $A_{f} \in G L(2, \mathbf{F})$ is a matrix representing a chain $A \in \Lambda$ then $[A]=\eta\left(\operatorname{det}\left(A_{f}\right)\right)$.

In [2] Kroll (cf. Subsection 1.6) defined order structures for Benz planes - and so also for Minkowski planes - starting inter alia from the notion of separation, i.e. a function which maps each quadruple $(a, b, c, d)$ consisting of concyclic points with $a, b \neq c, d$ on a value $[a, b \mid c, d]$ in $\{1,-1\}$ such that certain conditions are satisfied (cf. Sect. 1.5).

In Sect. 1.5 we recall for symmetric Minkowski planes $\mathfrak{M}$ the notions orthogonal valuation, order valuation and then halfordered and ordered symmetric Minkowski planes (M, []) (based on valuations). Theorem 1.13. describes properties of these structures and Theorem 1.14. recalls relations to the halforderings and orderings of the corresponding commutative field $(\mathbf{F},+, \cdot)$.

Following Kroll we introduce the concepts separation and order separation by conditions (T1), (T2) and (T1), (T2), (T3), respectively.

Theorem 2.1 shows how one can derive from an orthogonal valuation [] of a symmetric Minkowski plane $\mathfrak{M}$ a separation $\tau$ of $\mathfrak{M}$. The separation $\tau$ is harmonic iff the valuation [ ] is harmonic, and $\tau$ is an order separation iff [] is an order valuation.

Finally in Sect. 3 we start from a pair $(\mathfrak{M}, \tau)$ where $\mathfrak{M}$ is a symmetric Minkowski plane and $\tau$ a separation of $\mathfrak{M}$ and prove in Theorem 3.1 some properties of $(\mathfrak{M}, \tau)$. Then we show in Theorem 3.2 how one can derive from $\tau$ a halfordering $\eta_{\tau}$ for the corresponding field $(\mathbf{F},+, \cdot)$. Combining these results starting from a halfordered symmetric Minkowski ( $\mathfrak{M},[])$ we can derive firstly (as in [1]) a halfordering $\eta$ of the corresponding field $(\mathbf{F},+, \cdot)$ and secondly a separation $\tau$ of $\mathfrak{M}$ and then a halfordering $\eta_{\tau}$ of $(\mathbf{F},+, \cdot)$. We show $\eta=\eta_{\tau}$ and this proves our main result Theorem 3.3.

\section{Preliminaries and supplements}

\subsection{Hyperbola structures and Minkowski planes}

In this paper we will use mostly the same notations as in [1]. Let $\left(P, \mathfrak{G}_{1}, \mathfrak{G}_{2}\right)$ be a net. As in [1] let $P^{(3)}:=\left\{\{a, b, c\} \in\left(\begin{array}{c}P \\ 3\end{array}\right) \mid\{a, b, c\}\right.$ is joinable $\}$ and let $\mathfrak{C}$ be the set of all chains of $\left(P, \mathfrak{G}_{1}, \mathfrak{G}_{2}\right)$. The quadruple $\left(P, \mathfrak{C}, \mathfrak{G}_{1}, \mathfrak{G}_{2}\right)$ is then called maximal chain structure.

For $a, b, c, d \in P$ we add the notions $(a, b, c, d)^{\square}:=\{a b, b a, c d, d c\}$ and $\{a, b$, $c, d\}^{\square}:=\left\{\{a, b, c, d\},(a, b, c, d)^{\square},(a, c, d, b)^{\square},(a, d, b, c)^{\square}\right\}$. If $\{x, y, z, u\} \in\{a, b, c, d\}^{\square}$ then $\{x, y, z, u\}^{\square}=\{a, b, c, d\}^{\square}$.

A quadruple $\left(P, \Lambda, \mathfrak{G}_{1}, \mathfrak{G}_{2}\right)$ is called hyperbola structure if $\Lambda \subseteq \mathfrak{C}$ such that:

(I) $\forall\{a, b, c\} \in P^{(3)} \exists_{1} L \in \Lambda:\{a, b, c\} \subseteq L-$ we set $(a, b, c)^{\circ}:=L-$. 
If $\left(P, \Lambda, \mathfrak{G}_{1}, \mathfrak{G}_{2}\right)$ is a hyperbola structure then a subset $S$ of $P$ is called concyclic if there is a circle $L \in \Lambda$ such that $S \subseteq L-$ if $|S| \geq 3$ then $L$ is uniquely determined and we set then $S^{\circ}:=L-$. A hyperbola structure $\left(P, \Lambda, \mathfrak{G}_{1}, \mathfrak{G}_{2}\right)$ is called symmetric Minkowski plane if the so called "Symmetry Axiom" is satisfied which we can express in the following form (cf. [1, (2.7)]) :

(S) If a quadruple $(a, b, c, d)$ consists of concyclic points then also the set $(a, b, c, d)^{\square}$ is concyclic.

In a symmetric Minkowski plane, if $F:=\{a, b, c, d\}$ is a set consisting of four distinct concyclic points then by Axiom (S), the set $F^{\square}$ consists of four subsets of points and each subset consisting of four distinct concyclic points so that (by Axiom (I)) we can form the set

$$
\Lambda_{F}:=\left\{\{a, b, c, d\}^{\circ},(a, b, c, d)^{\square \circ},(a, c, d, b)^{\square \circ},(a, d, b, c)^{\square \circ}\right\}
$$

consisting of four distinct circles which are orthogonal in pairs. A quadruple $(a, b, c, d)$ consisting of four distinct concyclic points is called harmonic if $\{c, d\}=(a, b, c)^{\circ} \cap(a b, b a, c)^{\circ}$. Let $P^{(4 h)}$ denote the set of all harmonic quadruples.

From now on let $\mathfrak{M}=\left(P, \Lambda, \mathfrak{G}_{1}, \mathfrak{G}_{2}\right)$ be a symmetric Minkowski plane, let a circle $E \in \Lambda$ be fixed and for $A, B \in \mathfrak{C}$ let $A \cdot B:=t(A, E, B)=\widetilde{A B}(E)$ then (cf. $[1$, p. 118 f]):

Theorem 1.1. $(\mathfrak{C}, \cdot)$ is a group with the neutral element $E, t(A, B, C)=A$. $B^{-1} \cdot C$ and the orthogonality of chains is described by:

$$
A \perp B \Leftrightarrow A \cdot B^{-1}=B \cdot A^{-1} \text { and } A \neq B .
$$

Moreover $\Lambda$ is a subgroup of $(\mathfrak{C}, \cdot)$.

We recall that a quadruple $(A, B, C, D)$ of circles is called a quadrangle if $D=t(A, B, C)$, a quadrangle is called a square if $A \perp B \perp C \perp D \perp A$ and a total square if moreover $A \perp C$ and $B \perp D$. If $F$ is a set of four distinct concyclic points then $\Lambda_{F}$ is a total square,

\subsection{Properties of symmetric Minkowski planes}

In a symmetric Minkowski planes $\mathfrak{M}=\left(P, \Lambda, \mathfrak{G}_{1}, \mathfrak{G}_{2}\right)$ we define $\Gamma^{-}:=\{\widetilde{A B} \mid$ $A, B \in \Lambda\}$, denote by $\Gamma:=\left\langle\Gamma^{-}\right\rangle$the group generated by $\Gamma^{-}$and set

$$
\begin{aligned}
(\Lambda \times \Lambda)_{\perp} & :=\{(A, B) \in(\Lambda \times \Lambda) \mid A \perp B\} \text { and } \\
(\Lambda \times \Lambda)_{\perp, s}: & =\left\{(A, B) \in(\Lambda \times \Lambda)_{\perp} \mid A \cap B \neq \emptyset\right\} .
\end{aligned}
$$

For a set $S$ of concyclic points with $|S| \geq 3$ there is exactly one circle in $\Lambda$ containing $S$ which shall be denoted also by $S^{\circ}$.

Finally the notions of 1- and 2-perspectivities can be defined:

Let $A, B \in \Lambda$ then the map

$$
[A \stackrel{1}{\longrightarrow} B]: A \rightarrow B ; x \mapsto x B \text { and }[A \stackrel{2}{\longrightarrow} B]: A \rightarrow B ; x \mapsto B x
$$

is called 1- and 2-perspectivity, respectively. 
From [1, Section 2.1, (2.5),(2.6),(2.7)] follows:

Theorem 1.2. Let $A, B, C, X \in \Lambda$ and let $(a, b, c, d) \in X$ with $a, b \neq c, d$, then:

(1) $\Gamma \leqslant \operatorname{Aut}\left(P, \Lambda, \mathfrak{G}_{1} \cup \mathfrak{G}_{2}\right)$.

(2) If $a \neq b$ then $\{a b, b a, c d\} \in P^{(3)}, d c \in K:=(a b, b a, c d)^{\circ}$ and $K \perp X$.

(3) If $|\{a, b, c, d\}| \geqq 3$ then $X=(a, b, c, d)^{\circ}$ and $K:=\left((a, b, c, d)^{\square}\right)^{\circ}$ are uniquely determined circles of $\Lambda$ and $X \perp K$; if $a=b$ or $c=d$ then $K=\left((c, d, a)^{\beta}\right)^{\circ}$ and $a \in X \cap K$ or $K=\left((a, b, c)^{\beta}\right)^{\circ}$ and $c \in X \cap K$, respectively.

(4) If $|\{a, b, c, d\}|=4$ and $F:=\{a, b, c, d\} \subseteq E$ then $\Lambda_{F}$ is a subgroup of $(\Lambda, \cdot)$ and consists of four circles which are orthogonal in pairs (cf. Theorem 1.1), i.e. $\Lambda_{F}$ is a total square.

(5) If $A, B \perp C, A \cap C=B \cap C=\{p, q\}$ and $p \neq q$ then $A=B$.

Proof. (5) Assume $A \neq B$ hence $A \cap B=\{p, q\}$ then by [1, (3.5.1)], $p q, q p \in C$. But since $p, q \in C$ and $p \neq q$ we have $p q, q p \notin C$. Consequently $A=B$.

Theorem 1.3. Let $K \in \Lambda, a, b, c, d, e \in K$ with $a, b \neq c, d, e$ and $a \neq b$ and let $L_{c}:=\{a b, b a, d e\}^{\circ}, L_{d}:=\{a b, b a, e c\}^{\circ}, L_{e}:=\{a b, b a, c d\}^{\circ}$ and $M:=$ $\widetilde{L_{e} L_{c}}\left(L_{d}\right)=L_{e} \cdot L_{d}^{-1} \cdot L_{c}$ then $M=\{a b, b a, d\}^{\circ}, M \perp K$ and $d \in M \cap K$, i.e. $(M, K) \in(\Lambda \times \Lambda)_{\perp s}$.

Proof. Since $\{a b, b a, c d, d c\} \subseteq L_{e},\{a b, b a, c e, e c\} \subseteq L_{d}$ and $\{a b, b a, d e, e d\} \subseteq$ $L_{c}$ we have $a b, b a \in F i x \widetilde{L_{e} L_{c}}$ and $\widetilde{L_{e} L_{c}}(c e)=(d e)(c d)=d$ hence $M=$ $\widetilde{L_{e} L_{c}}\left(L_{d}\right)=\{a b, b a, d\}^{\circ}$ with $M \perp K$ and $d \in M \cap K$ and so $(M, K) \in$ $(\Lambda \times \Lambda)_{\perp s}$.

By $[1,(2.5 .2)$ and (3.9)] we have:

Theorem 1.4. The set $\Gamma^{-}:=\{\widetilde{A B} \mid A, B \in \Lambda\}$ consists of automorphisms of $(P, \Lambda)$ and acts transitively on the set of circles $\Lambda$ preserving orthogonality and if $\gamma$ is an element of the group $\Gamma$ generated by $\Gamma^{-}$then there exist exactly two elements $A, B \in \Lambda$ such that either $\gamma=\widetilde{A B}$ or $\gamma=\widetilde{A B} \circ \widetilde{E}$. Moreover $\widetilde{A B} \circ \widetilde{C D}=\left(A \cdot \widetilde{\left.D^{-1}\right)\left(C^{-1}\right.} \cdot B\right) \circ \widetilde{E}$ and $\Gamma^{+}:=\widetilde{E} \circ \Gamma^{-}$acts regularly on the set $P^{(3)}$.

Therefore, if $A, B \in \Lambda$ and $C:=\widetilde{A E}(B)=A \cdot B^{-1} \cdot E=A \cdot B^{-1}$ we have:

$$
(A, B) \in(\Lambda \times \Lambda)_{\perp, s} \Leftrightarrow(E, C) \in(\Lambda \times \Lambda)_{\perp, s} .
$$

Consequently, $\forall(A, B),(C, D) \in(\Lambda \times \Lambda)_{\perp, s}:|A \cap B|=|C \cap D|$.

\subsection{The corresponding field and the characteristic of a symmetric Minkowski plane}

As in [1] we denote by $(\mathbf{F},+, \cdot)$ the commutative field corresponding to the symmetric Minkowski plane $\mathfrak{M}$ (cf. [1, sec.1]) and we define char $\mathfrak{M}:=\operatorname{char} \mathbf{F}$. If $\left(\begin{array}{l}c_{1}, c_{2} \\ c_{3}, c_{4}\end{array}\right) \in G L(2, \mathbf{F})$ represents the circle $C$ then $C \perp E$ implies $c_{1}=-c_{4}$ and if $c_{1}=-c_{4}$ then: 
$C \cap E \neq \emptyset \Leftrightarrow$ the characteristic equation $\Xi_{C}(t)=t^{2}-\left(c_{1}^{2}+c_{2} \cdot c_{3}\right)=0$ is solvable $\Leftrightarrow\left(c_{1}^{2}+c_{2} \cdot c_{3}\right) \in \mathbf{F}^{(2)}:=\left\{\lambda^{2} \mid \lambda \in \mathbf{F} \backslash\{0\}\right\}$.

Hence if $\left(c_{1}^{2}+c_{2} \cdot c_{3}\right) \in \mathbf{F}^{(2)}$ then: $|C \cap E|=2$ if $\operatorname{char} \mathbf{F} \neq 2$ and $|C \cap E|=1$ if $\operatorname{char} \mathbf{F}=2$. This supplements the statements of $[1,(3.7)]$ :

Theorem 1.5. Let $(A, B) \in(\Lambda \times \Lambda)_{\perp, s}$ then:

(1) $|A \cap B|=2 \Leftrightarrow \operatorname{char} \mathfrak{M} \neq 2$.

(2) $|A \cap B|=1 \Leftrightarrow \operatorname{char} \mathfrak{M}=2$.

(3) If char $\mathfrak{M} \neq 2$ then $\forall(a, b, c) \in P^{(3)} \exists_{1} d \in P$ such that $(a, b, c, d)$ is a harmonic quadruple and $d$ is given by $\{a, b, c\}^{\circ} \cap\{a b, b a, c\}^{\circ}=\{c, d\}$. If char $\mathfrak{M}=2$, there are no harmonic quadruples.

Now let $C, D \in \Lambda$ be circles with $C, D \perp E$ and $C \cap E \neq \emptyset, D \cap E \neq \emptyset$ and let $\left(\begin{array}{c}c_{1}, c_{2} \\ c_{3},-c_{1}\end{array}\right),\left(\begin{array}{c}d_{1}, d_{2} \\ d_{3},-d_{1}\end{array}\right) \in G L(2, \mathbf{F})$ be a representing matrices. Then we may assume $d_{1}^{2}+d_{2} \cdot d_{3}=c_{1}^{2}+c_{2} \cdot c_{3}=1$ and moreover $c_{1}=1$ if $c_{2} \cdot c_{3}=0$ and $d_{1}=1$ if $d_{2} \cdot d_{3}=0$. Let $M_{C}:=\left(\begin{array}{c}0, c_{2} \\ 1,-c_{1}\end{array}\right)$ if $c_{2} \neq 0, M_{C}:=\left(\begin{array}{l}c_{1}, 1 \\ c_{3}, 0\end{array}\right)$ if $c_{2}=0 \neq c_{3}$ and $M_{C}:=\left(\begin{array}{c}1,1 \\ 1,-1\end{array}\right)$ if $c_{2}=c_{3}=0$ and $\operatorname{char} \mathbf{F} \neq 2$ and let $M_{D}$ be defined accordingly. Then if $L \in \Lambda$ is the circle represented by the matrix $M_{D} \cdot M_{C}^{-1}$. Then the map $\widetilde{L L^{-1}}$ fixes $E$ and maps $C$ onto $D$. This gives us:

Theorem 1.6. $\Gamma$ acts transitively on the set $(\Lambda \times \Lambda)_{\perp s}$.

In the same way using representing matrices one confirms the Three Reflection Theorems:

Theorem 1.7. Let $A, B, C \in \Lambda$ with $A \cap B \cap C \neq \emptyset$ and $p \in A \cap B \cap C$ then:

(1) If $|A \cap B \cap C| \geq 2$ then: $\exists D \in \Lambda: \widetilde{A} \circ \widetilde{B} \circ \widetilde{C}=\widetilde{D}$.

(2) If there is an $\bar{L} \in \Lambda$ with $p \in L$ and $A, B, C \perp L$ then: $\exists D \in \Lambda$ : $\widetilde{A} \circ \widetilde{B} \circ \widetilde{C}=\widetilde{D}($ and then $p \in D$ and $D \perp L$ or $D=L)$.

By the definition of the maps $\widetilde{A B}$ one obtains the following representations of 1- and 2-perspectivities:

Proposition 1.8. Let $A, B, C \in \Lambda$ and $D:=\widetilde{A B}(C)$ then:

(1) $[A \stackrel{1}{\longrightarrow} B]=\widetilde{B A}_{\mid A}$ and $[A \stackrel{2}{\longrightarrow} B]=\widetilde{A B}{ }_{\mid A}$.

(2) $\widetilde{A B}_{\mid C}=[A \stackrel{2}{\longrightarrow} D] \circ[C \stackrel{1}{\longrightarrow} A]=[B \stackrel{1}{\longrightarrow} D] \circ[C \stackrel{2}{\longrightarrow} B]$.

(3) $\bar{A}: E \rightarrow E ; x \mapsto E(x A)=[A \stackrel{2}{\longrightarrow} E] \circ[E \stackrel{1}{\longrightarrow} A](x)=\widetilde{A E} \circ \widetilde{A E}(x)$.

(4) If $A \perp B$ then $\psi:=[B \stackrel{2}{\longrightarrow} A] \circ[A \stackrel{1}{\longrightarrow} B]=\widetilde{B A} \circ \widetilde{B A}_{\mid A}$ and if for $x \in A, x^{\prime}:=\widetilde{B}(x)$ then:

$$
x^{\prime}=\psi(x) \in A,\left(x^{\prime}\right)^{\prime}=x, x x^{\prime} \in B \quad \text { and } \quad \widetilde{A}\left(x x^{\prime}\right)=x^{\prime} x \in B .
$$

Theorem 1.9. Let $(a, b, c, d)$ and $\left(a^{\prime}, b^{\prime}, c^{\prime}, d^{\prime}\right)$ be harmonic quadruples and let $\gamma \in \Gamma$ then: 
(1) $(b, a, c, d),(c, d, a, b),(a b, b a, c d, d c),(a b, b a, c, d)$ and $(\gamma(a), \gamma(b), \gamma(c), \gamma(d))$ are harmonic quadruples.

(2) $\exists \sigma \in \Gamma: \sigma(a)=a^{\prime}, \sigma(b)=b^{\prime}, \sigma(c)=c^{\prime}, \sigma(d)=d^{\prime}$.

(3) The circles $K:=\{a, b, c\}^{\circ}, L:=\{a b, b a, c d\}^{\circ}, M:=\{a b, b a, c\}^{\circ}$ and $N:=\{a, b, c d\}^{\circ}$ are orthogonal in pairs hence $\mathfrak{H}:=(K, L, M, N)$ is a total square in $\Lambda$ and $(K, M),(K, N),(L, M),(L, N) \in(\Lambda \times \Lambda)_{\perp, s}$ and $K \cap M=\{c, d\}, K \cap N=\{a, b\}, L \cap M=\{a b, b a\}$ and $L \cap N=\{c d, d c\}$. $K^{-1} \cdot\{K, L, M, N\}$ is a subgroup of $(\Lambda, \cdot)$ isomorphic to the Klein four group.

Proof. (1) The definition harmonic is symmetric in the first two and in the last two arguments and from $\{c, d\}=\{a, b, c\}^{\circ} \cap\{a b, b a, c\}^{\circ}$ we obtain $\{a, b, c\}^{\circ}=$ $\left(\{c, d, a\}^{\circ}\right.$ and $a b, b a, c, d$ are four distinct concyclic points hence by $\mathbf{( S )},(a b, b a$, $c, d)^{\square}=\{a, b, c d, d c\}$ is concyclic, i.e. $\{c d, d c, a\}^{\circ} \ni a, b$ and so $\{a, b\}=$ $\{c, d, a\}^{\circ} \cap\{c d, d c, a\}^{\circ}$, i.e. $(c, d, a, b) \in P^{(4 h)}$.

(2) By Theorem 1.6 there is exactly one $\sigma \in \Gamma^{+}$with $\sigma(a)=a^{\prime}, \sigma(b)=$ $b^{\prime}, \sigma(c)=c^{\prime}$ and so $\{\sigma(c), \sigma(d)\}=\{\sigma(a), \sigma(b), \sigma(c)\}^{\circ} \cap\{\sigma(a) \sigma(b), \sigma(b) \sigma(a)$, $\sigma(c)\}^{\circ}=\left\{a^{\prime}, b^{\prime}, c^{\prime}\right\}^{\circ} \cap\left\{a^{\prime} b^{\prime}, b^{\prime} a^{\prime}, c^{\prime}\right\}^{\circ}=\left\{c^{\prime}, d^{\prime}\right\}$ implying $\sigma(d)=d^{\prime}$.

Theorem 1.10. Let $a, b, c, d$ be four distinct concyclic points, $K:=\{a, b, c\}^{\circ}$, $C:=\{b c, c b, a\}^{\circ}, D:=\{b d, d b, a\}^{\circ}, H:=\{c d, d c, a\}^{\circ}$ and let $B \in \Lambda$ [according to Theorem $1.7(2)]$ be such that $\widetilde{B}=\widetilde{D} \circ \widetilde{H} \circ \widetilde{C}$. Then $\widetilde{B}(\widetilde{C}(d))=\widetilde{D}(c)$ and $(a, b, \widetilde{C}(d), \widetilde{D}(c))$ is harmonic.

Proof. By the definitions we have: $a \in C, H, D, K ; C, H, D \perp K, \widetilde{C}(b)=c$, $\widetilde{H}(c)=d$ and $\widetilde{D}(d)=b$ hence, by Theorem $1.9(2), B \perp K, \widetilde{B}(a)=\widetilde{D} \circ \widetilde{H} \circ$ $\widetilde{C}(a)=a, \widetilde{B}(b)=\widetilde{D} \circ \widetilde{H} \circ \widetilde{C}(b)=b$ and $\widetilde{B}(\widetilde{C}(d))=\widetilde{D} \circ \widetilde{H} \circ \widetilde{C}(\widetilde{C}(d))=$ $\widetilde{D} \circ \widetilde{H}(d)=\widetilde{D}(c)$. Since moreover $\widetilde{C}(d), \widetilde{D}(c) \in K$ we have $\{\widetilde{C}(d), \widetilde{D}(c)\}=$ $\{a, b, \widetilde{C}(d)\}^{\circ} \cap\{a b, b a, \widetilde{C}(d)\}^{\circ}$ i.e. $(a, b, \widetilde{C}(d), \widetilde{D}(c))$ is harmonic.

\subsection{Orthogonal valuations and separations in symmetric Minkowski planes}

In $[1$, p. 120 and p. 126] a map [ ] : $\Lambda \rightarrow\{1,-1\} ; X \mapsto[X]$ was called orthogonal valuation if

(0 1) For each square $(A, B, C, D):[A] \cdot[B] \cdot[C] \cdot[D]=1$. and homomorphic valuation if this equation holds true even for each quadrangle. An orthogonal valuation is called order valuation if

(0 2) For each total square $(A, B, C, D)$ : exactly two of the values $[A],[B]$, $[C],[D]$ equal 1.

By Theorems 1.4, 1.5 and $[1,(4.5)$ and (4.8)] we have:

Theorem 1.11. Let [ ] : $\Lambda \rightarrow\{1,-1\} ; X \mapsto[X]$ be an orthogonal valuation of $\mathfrak{M}$, let $E \in \Lambda$ with $[E]=1$ be fixed, for $A, B \in \Lambda$ let $A \cdot B:=t(A, E, B)$ and for $\gamma=\widetilde{A B} \in \Gamma^{-}$or $\gamma=\widetilde{A B} \circ \widetilde{E} \in \Gamma^{+}$let $[\gamma]^{\prime}:=[A] \cdot[B]$. Then: 
(1) [ ] is a homomorphic valuation hence $\forall A, B, C \in \Lambda:[t(A, B, C)]=[A$. $\left.B^{-1} \cdot C\right]=[A] \cdot[B] \cdot[C]$ and $[A \cdot B]=[A] \cdot[B]$.

(2) []$^{\prime}:(\Gamma, \circ) \rightarrow(\{1,-1\}, \cdot) ; \gamma \mapsto[\gamma]^{\prime}$ is a homomorphism.

(3) $\forall X \in \Lambda, \forall \gamma \in \Gamma:[\gamma(X)]=[\gamma]^{\prime} \cdot[X]$.

(4) There is an $\iota \in\{1,-1\}$ such that: $\forall(A, B) \in(\Lambda \times \Lambda)_{\perp s}:[A] \cdot[B]=\iota$.

(5) If [ ] is an order valuation then $\iota=-1$.

An orthogonal valuation [ ] is called harmonic if $\iota=-1$ and anharmonic if $\iota=1$.

Let $P^{(4)}:=\left\{(a, b, c, d) \in P^{4} \mid\{a, b, c, d\}\right.$ concyclic $\left.\wedge a, b \neq c, d\right\}$.

A map $\tau: P^{(4)} \rightarrow(\{1,-1\}, \cdot) ;(a, b, c, d) \mapsto[a, b \mid c, d]$ is called a separation if the following conditions are satisfied:

(T1) For all concyclic $a, b, c, d, e \in P$ with $a, b \neq c, d, e$ holds:

$$
[a, b \mid c, d] \cdot[a, b \mid d, e]=[a, b \mid c, e] .
$$

(T2) For all $(a, b, c, d) \in P^{(4)}$, for all $L \in \Lambda$ : $[a, b \mid c, d]=[L a, L b \mid L c, L d]=[a L, b L \mid c L, d L]$, i.e. the separation function is invariant by 1- and 2-perspectivities.

If moreover the axiom

(T3) $\forall(a, b, c, d) \in P^{(4)}$ with $a \neq b, c \neq d$ : exactly one of the values $[a, b \mid c, d]$, $[a, c \mid d, b],[a, d \mid b, c]$ is -1

is valid then $\tau$ is called an order separation.

A separation is called harmonic if for a harmonic quadruple $(a, b, c, d)$ (and then for all) the value $[a, b \mid c, d]$ is -1 . This definition corresponds E. Sperner's definition of a harmonic separation.

\subsection{Kroll's order concepts for Minkowski planes}

Kroll [2] developed a thoroughly theory of order questions for Benz planes. Benz planes contain as a subclass the Minkowski planes. We present some of Kroll's concepts specialized for Minkowski planes $\mathfrak{M}:=\left(P, \Lambda, \mathfrak{G}_{1}, \mathfrak{G}_{2}\right)$ : Let $(\Lambda \times P \times P)^{*}:=\{(A, b, c) \in \Lambda \times P \times P \mid b, c \notin A\}$. Then a map $\alpha:(\Lambda \times P \times P)^{*} \rightarrow$ $\{1,-1\} ;(A, b, c) \mapsto(A \mid b, c)$ is called order function of $\mathfrak{M}$ if :

$01 \forall A \in \Lambda$ and $\forall x, y, z \in P \backslash A:(A \mid x, y) \cdot(A \mid y, z) \cdot(A \mid z, x)=1$.

02 (circle relation) $\forall A, B, C \in \Lambda$ such that $A \cap C=B \cap C$ and $|A \cap C| \geq 1$ and $\forall x, y \in C \backslash A:(A \mid x, y)=(B \mid x, y$,$) .$

OV $\forall G \in \mathfrak{G}_{1} \cup \mathfrak{G}_{2}, \forall A, B \in \Lambda$ with $A \cap G=B \cap G$ and $\forall c, d \in G \backslash A$ : $(A \mid c, d)=(B \mid c, d)$ (cf. [2, p. 224 and 231]).

If $(\mathfrak{M}, \alpha)$ is a Minkowski plane with an order function, Kroll derives a separation $\tau_{\alpha}$ by:

(V) For $K \in \Lambda$, for $a, b, c, d \in K$ with $a, b \neq c, d$ and $A, L \in \Lambda$ with $A \cap K=$ $\{a\}, L \cap K=\{a, b\}$ let $[a, b \mid c, d]:=(A \mid c, d) \cdot(L \mid c, d) .([2$, p. 234]) 
He shows that $\tau_{\alpha}$ satisfies the conditions (T1) and (T2) if $\mathfrak{M}$ is a symmetric Minkowski plane (cf. [2, p. 234 and p. 237], T4*i). Conversely every separation of a symmetric Minkowski plane is a separation in the sense of Kroll.

\section{From an orthogonal valuation to a separation}

Theorem 2.1. Let (M, [ ]) be a halfordered symmetric Minkowski plane, let $\iota$ be the value corresponding to $(\mathfrak{M},[])$ according to Theorem 1.11(4) and let $\tau: P^{(4)} \rightarrow\{1,-1\} ;(a, b, c, d) \mapsto[a, b \mid c, d]$ be the map defined by $[a, b \mid c, d]:=1$ if $a=b$ and $[a, b \mid c, d]:=\iota \cdot\left[\{a, b, c, d\}^{\circ}\right] \cdot\left[(a, b, c, d)^{\square \circ}\right]$ if $a \neq b$ then:

(1) $\tau$ is a separation and if $(a, b, c, d)$ is harmonic then $[a, b \mid c, d]=\iota$. Hence the derived separation $\tau$ is harmonic if and only if the valuation [ ] is harmonic.

(2) If [ ] is an order valuation then $\tau$ is an order separation.

Proof. (T1) Let $(a, b, c, d),(a, b, d, e) \in P^{(4)}$. If $a=b$ then $[a, b \mid c, d]=[a, b \mid d, e]$ $=[a, b \mid c, e]=1$ and the equation (T1) is valid. Let $a \neq b$ then $(a, b, c),(a, b, d) \in$ $P^{(3)}$, so we can form the circles $K:=\{a, b, c\}^{\circ}$ and $K^{\prime}:=\{a, b, d\}^{\circ}$ and by the assumption, $(a, b, c, d),(a, b, d, e) \in P^{(4)}$, we have $a, b, c, d, e \in K=K^{\prime}$. If we define the circles $L_{c}, L_{d}, L_{e}$ and $M$ according to Theorem 1.3 then $(M, K) \in$ $(\Lambda \times \Lambda)_{\perp s}$ hence by Theorem 1.11(4), $[M] \cdot[K]=\iota$ and by definition of $\tau$ we have $[a, b \mid c, d]:=\iota \cdot[K] \cdot\left[L_{e}\right],[a, b \mid d, e]:=\iota \cdot[K] \cdot\left[L_{c}\right]$ and $[a, b \mid e, c]:=\iota \cdot[K] \cdot\left[L_{d}\right]$. By Theorem 1.11(1)), this implies:

$[a, b \mid c, d] \cdot[a, b \mid d, e] \cdot[a, b \mid e, c]=\iota \cdot[K] \cdot\left[L_{e}\right] \cdot \iota \cdot[K] \cdot\left[L_{c}\right] \cdot \iota \cdot[K] \cdot\left[L_{d}\right]=$ $\iota \cdot[K] \cdot\left[L_{e} \cdot L_{d}^{-1} \cdot L_{c}\right]=\iota \cdot[K] \cdot[M]=\iota \cdot \iota=1$.

(T2) Let $K, L \in \Lambda,\{a, b, c, d\} \in\left(\begin{array}{c}K \\ 4\end{array}\right)$ and $H:=\{a b, b a, c d\}^{\circ}$ then by Theorem 1.2(2), $H \perp K$ hence $[a, b \mid c, d]=[K] \cdot[H] \cdot \iota$ and by Proposition 1.8,

$$
\pi_{1}:=[K \stackrel{1}{\longrightarrow} L]=\widetilde{L K}_{\mid K}, \quad \pi_{2}:=[K \stackrel{2}{\longrightarrow} L]=\widetilde{K L}_{\mid K} .
$$

Then $\widetilde{K L}(K)=L=\widetilde{L K}(K)$ and if $H_{1}:=\widetilde{L K}(H), H_{2}:=\widetilde{K L}(H)$ then $H_{1}, H_{2} \perp L$ and so $H_{i}=\left(\left(\pi_{i}(a), \pi_{i}(b), \pi_{i}(c), \pi_{i}(d)\right)^{\square}\right)^{\circ}$. Thus

$\left[\pi_{i}(a), \pi_{i}(b) \mid \pi_{i}(c), \pi_{i}(d)\right]=[L] \cdot\left[H_{i}\right] \cdot \iota$ with $H_{1}=L \cdot H^{-1} \cdot K, H_{2}=K \cdot$ $H^{-1} \cdot L$ implying by Theorem 1.11(1), $\left[H_{i}\right]=[K] \cdot[H] \cdot[L]$. Therefore $\iota$. $\left[\pi_{i}(a), \pi_{i}(b) \mid \pi_{i}(c), \pi_{i}(d)\right]=[K] \cdot[H] \cdot[L] \cdot[L]=[K] \cdot[H]=\iota \cdot[a, b \mid c, d]$.

Now let $(a, b, c, d)$ be harmonic and let $K, L, M, N$ be defined according to Theorem 1.9(3). Then by Theorem 1.9(3) and Theorem 1.11(4), $[a, b \mid c, d]=$ $[K] \cdot[L] \cdot \iota=[K] \cdot[M] \cdot[M] \cdot[L] \cdot \iota=\iota \cdot \iota \cdot \iota=\iota$.

(2) By Theorem 1.11(5), $\iota=-1$. Therefore using the notations of Theorem $1.2(3)$ we have:

$$
[a, b \mid c, d]=-[X] \cdot[Y], \quad[a, c \mid d, b]=-[X] \cdot[Z], \quad[a, d \mid b, c]=-[X] \cdot[U],
$$


by Theorem 1.2(4) the quadruple $(X, Y, Z, U)$ is a total square and so by (02), exactly two of the values $[X],[Y],[Z],[U]$ are 1 . But from this observations follows (T3).

\section{From a separation to a halfordering of the corresponding field}

We start from the assumption that the Minkowski plane $\mathfrak{M}$ is provided with a separation $\tau$.

Theorem 3.1. Let $(a, b, c, d) \in P^{(4)}$ with $a \neq b ; c \neq d$ and let $\gamma \in \Gamma$ then:

1. $[\gamma(a), \gamma(b) \mid \gamma(c), \gamma(d)]=[a, b \mid c, d]$.

2. $[a, b \mid c, d] \cdot[a, c \mid d, b] \cdot[a, d \mid b, c]=\left[a^{\prime}, b^{\prime} \mid c^{\prime}, d^{\prime}\right]$ where $\left(a^{\prime}, b^{\prime}, c^{\prime}, d^{\prime}\right)$ is any harmonic point quadruple.

Proof. (1) Follows from Proposition 1.8 and (T2).

(2) Let $C:=\{b c, c b, a\}^{\circ}$ and $D:=\{b d, d b, a\}^{\circ}$. Then $\widetilde{C}(a)=a, \widetilde{C}(b)=$ $c, \widetilde{C}(c)=b$ and $\widetilde{D}(a)=a, \widetilde{D}(b)=d$ and so by $(1),[a, c \mid d, b]=[a, b \mid \widetilde{C}(d), c]$ and $[a, d \mid b, c]=[a, b \mid d, \widetilde{D}(c)]$. By $(\mathrm{T} 1)$ we have $[a, b \mid c, d] \cdot[a, c \mid d, b] \cdot[a, d \mid b, c]=$ $[a, b \mid c, d] \cdot[a, b \mid \widetilde{C}(d), c] \cdot[a, b \mid d, \widetilde{D}(c)]=[a, b \mid d, \widetilde{C}(d)] \cdot[a, b \mid d, \widetilde{D}(c)]=[a, b \mid \widetilde{C}(d)$, $\widetilde{D}(c)]$. By Theorem 1.10, $[a, b \mid \widetilde{C}(d), \widetilde{D}(c)]$ is a harmonic quadruple. By Theorem 1.9 and (T2) the $\tau$-value for all harmonic quadruples is the same.

We recall that the commutative field $(\mathbf{F},+, \cdot)$ corresponding to the symmetric Minkowski plane $\mathfrak{M}$ can be obtained as follows:

Theorem 3.2. Let $0,1, \infty$ be three distinct points of a fixed circle $E \in \Lambda$ and let $\mathbf{F}:=E \backslash\{\infty\}$. For $a, b \in \mathbf{F}$ and $c \in \mathbf{F}^{*}:=\mathbf{F} \backslash\{0\}$ let $A^{+}, C \cdot \Lambda$ be circles determined by: $A^{+}:=E$ if $a=0,0 a \in A^{+}$and $A^{+} \cap E=\{\infty\}$ if $a \neq 0, C:=\{0, \infty, 1 c\}^{\circ}$ and let $\overline{A^{+}}:=\widetilde{A^{+} E} \circ \widetilde{A^{+} E} \mid \overline{C^{*}}:=\widetilde{C^{\cdot} E} \circ \widetilde{C^{\cdot} E_{\mid E}}$ [cf. Proposition 1.8(3)], $a+b:=\overline{A^{+}}(b), c \cdot b:=\overline{C \cdot}(b)$. Then:

(1) $(\mathbf{F},+, \cdot)$ is a commutative field.

(2) The function $\eta: \mathbf{F}^{*} \rightarrow\{1,-1\} ; x \mapsto[0, \infty \mid 1, x]$ induced by the separation $\tau$ is a halfordering of $(\mathbf{F},+, \cdot)$.

(3) If $\tau$ is an order separation then $\eta$ is an ordering of $(\mathbf{F},+, \cdot)$.

Proof. (2) Let $a, b \in \mathbf{F}^{*}$. By the definition of $\overline{A^{*}}, \overline{A^{*}}(0)=0, \overline{A^{+}}(\infty)=\infty$, $\bar{A}(1)=a$ and $\bar{A}(b)=a \cdot b$ and so by Theorem 3.1, $[0, \infty \mid 1, b]=[0, \infty \mid a, a \cdot b]$ hence together with (T1), $\eta(a \cdot b)=[0, \infty \mid 1, a \cdot b]=[0, \infty \mid 1, a] \cdot[0, \infty \mid a, a \cdot b]=$ $[0, \infty \mid 1, a] \cdot[0, \infty \mid 1, b]=\eta(a) \cdot \eta(b)$.

Since $\widetilde{A^{+} E}(\infty)=\infty$ we have by Theorem 3.1, [ $[, x \mid y, z]=[\infty, a+x \mid a+y, a+z]$ for $x, y, z \in \mathbf{F}$. Now let $a \neq 0,1$. Then by using this formula and (T2), 


$$
\begin{aligned}
& {[\infty, 0 \mid 1, a]=\eta(a)} \\
& {[\infty, 1 \mid a, 0]=[\infty, 0 \mid a-1,-1]=[\infty, 0 \mid 1,-1] \cdot[\infty, 0 \mid 1, a-1]} \\
& \quad=\eta(-1) \cdot \eta(a-1)=\eta(1-a) \text { and } \\
& {[\infty, a \mid 0,1]=[\infty, 0 \mid-a, 1-a]=[\infty, 0 \mid 1,-a] \cdot[\infty, 0 \mid 1,1-a]} \\
& \quad=\eta(-a) \cdot \eta(1-a)=\eta\left(a^{2}-a\right)=\eta(a) \cdot \eta(a-1) .
\end{aligned}
$$

(3) If $\tau$ is an order separation then exactly one of these three values is equal -1 . Hence if $\eta(a)=1$ then $-\eta(1-a)=\eta(a-1)=\eta((-1) \cdot((1-a))=$ $\eta(-1) \cdot \eta(1-a)$ thus $\eta(-1)=-1$ and so $\eta(-a)=\eta((-1) \cdot a)=-\eta(a)$. Now let $x, y \in \mathbf{F}^{*}$ with $\eta(x)=\eta(y)=1$. For $a:=-x$ we obtain $\eta(-x)=-1$ and so $\eta(1-a)=\eta(1+x)=1$. By $\eta\left(x^{-1} \cdot y\right)=(\eta(x))^{-1} \cdot \eta(y)=1$, this implies $\eta(x+y)=\eta\left(x \cdot\left(1+x^{-1} y\right)=\eta(x) \cdot \eta\left(1+x^{-1} y\right)=1\right.$ and this is the monotony law for the addition.

Starting from a symmetric Minkowski plane $\mathfrak{M}$ endowed with an orthogonal valuation [ ], we derived a separtion $\tau$ for $\mathfrak{M}$ via Theorem 2.1 and from the separation $\tau$, a halfordering $\eta_{1}$ for the corresponding field $(\mathbf{F},+, \cdot)$ via Theorem 3.2. We show that $\eta_{1}$ and the halfordering $\eta_{2}$ derived from $(\mathfrak{M},[])$ via [1](4.7) are equal. Let $x \in \mathbf{F}^{*}$ and $X:=\{0 \infty, \infty 0,1 x\}^{\circ}$. Then $X \perp E$, by Theorem 1.11(1), $[E]=1$ and so $\eta_{1}(x)=[0, \infty \mid 1, x]=[E] \cdot[X] \cdot \iota=[X] \cdot \iota$ and moreover, $\left(\begin{array}{ll}0 & 1 \\ x & 0\end{array}\right)$ is a matrix representing $X$. Therefore by $[1](4.7),[X]=\eta_{2}\left(\operatorname{det}\left(\left(\begin{array}{ll}0 & 1 \\ x & 0\end{array}\right)\right)=\eta_{2}(-x)=\right.$ $\eta_{2}(-1) \cdot \eta_{2}(x)$ hence $\eta_{1}(x)=\iota \cdot \eta_{2}(-1) \cdot \eta_{2}(x)$. The circles $E$ and $I$ represented by the matrices $\left(\begin{array}{ll}1 & 0 \\ 0 & 1\end{array}\right)$ and $\left(\begin{array}{ll}0 & 1 \\ 1 & 0\end{array}\right)$ (with the determinants 1 and -1 ) are orthogonal and containing the point 1 . Hence $(E, I) \in(\Lambda \times \Lambda)_{\perp s}$ and so by Theorem $1.11(4), \iota=[E] \cdot[I]=\eta_{2}(1) \cdot \eta_{2}(-1)=\eta_{2}(-1)$ and so $\eta_{1}(x)=\eta_{2}(x)$ for all $x \in \mathbf{F}^{*}$. This gives us our main result:

Theorem 3.3. Let $\mathfrak{M}$ be a symmetric Minkowski plane and let $(\mathbf{F},+, \cdot)$ be the corresponding commutative field. Then there are a one to one correspondences between the orthogonal valuations of $\mathfrak{M}$, the separations of $\mathfrak{M}$ and the halforderings of $(\mathbf{F},+, \cdot)$ where the order valuations correspond with the order separations and with the orderings of the field.

Open Access. This article is distributed under the terms of the Creative Commons Attribution Noncommercial License which permits any noncommercial use, distribution, and reproduction in any medium, provided the original author(s) and source are credited.

\section{References}

[1] Karzel, H., Kosiorek, J., Matraś, A.: Ordered symmetric Minkowski planes I. J. Geom. 93, 116-127 (2009)

[2] Kroll, H.-J.: Anordnungsfragen in Benz-Ebenen. Abh. Math. Semin. Univ. Hambg. 46, 217-255 (1977) 
Helmut Karzel

Zentrum Mathematik

T.U. München

80290 Munich

Germany

e-mail: karzel@ma.tum.de

Jarosław Kosiorek and Andrzej Matraś

Faculty of Mathematics and Computer Science

University of Warmia and Mazury in Olsztyn

Żolnierska 14

10-561 Olsztyn

Poland

e-mail: kosiorek@matman.uwm.edu.pl

e-mail: matras@uwm.edu.pl

Received: March 13, 2006.

Revised: December 17, 2009. 